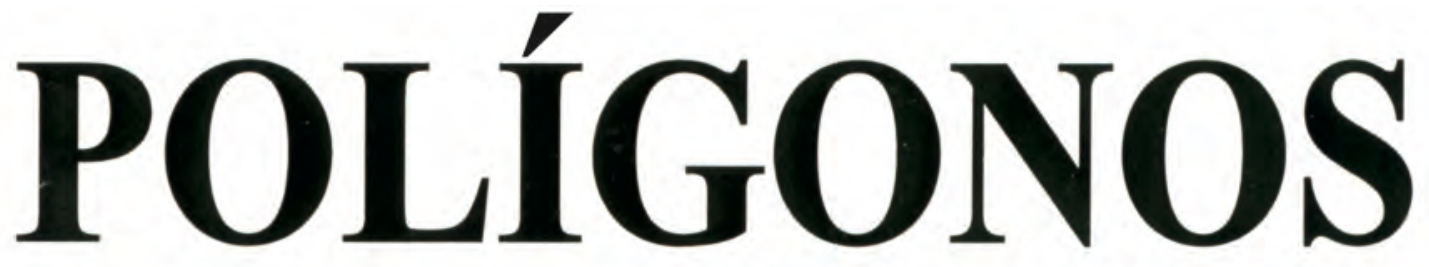

REVISTA DE GEOGRAFIA

2013

\title{
Segunda época
}

24

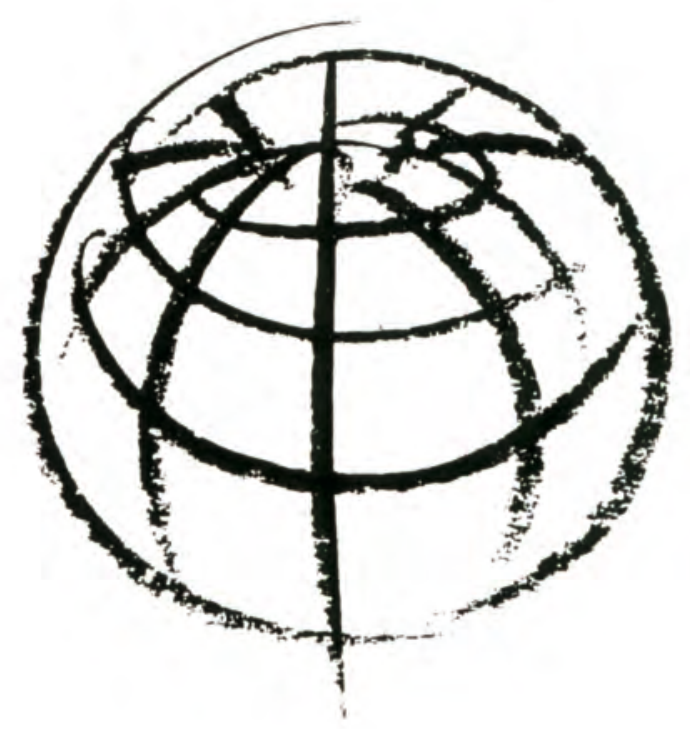

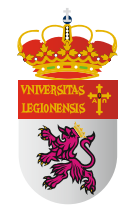

Universidad de León

Departamento de Geografía y Geología
Universidad de Salamanca

Departamento de Geografía

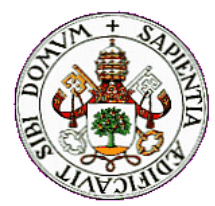

Universidad de Valladolid Departamento de Geografía 


\title{
POLÍGONOS
}

Revista de Geografía

\author{
CONSEJO DE REDACCIÓN \\ DIRECTORES \\ Guillermo Calonge Cano (Univ. de Valladolid) \\ Lorenzo López Trigal (Univ. de León) \\ Juan Ignacio Plaza Gutiérrez (Univ. de Salamanca) \\ SECRETARIO \\ José Cortizo Álvarez (Univ. de León) \\ VOCALES \\ Eugenio Baraja Rodríguez (Univ. de Valladolid) \\ Antonio Ceballos Barbancho (Univ. de Salamanca) \\ Carmen Delgado Viñas (Univ. de Cantabria) \\ Raquel Domínguez Fernández (Univ. de León) \\ Felipe Fernández García (Univ. de Oviedo) \\ Casildo Ferreras Chasco (Univ. Complutense de Madrid) \\ José Luis García Cuesta (Univ. de Valladolid) \\ Rubén Camilo Lois González (Univ. de Santiago) \\ David Ramos Pérez (Univ. de Salamanca)
}

\section{CONSEJO ASESOR}

Dr. Angel Cabo Alonso. Universidad de Salamanca Dra. Carminda Cavaco. Universidad de Lisboa

Dr. Pablo José Cicolella. Universidad de Buenos Aires

Dr. Pedro Cunill Grau. Universidad Central de Venezuela

Dr. Michael Drain. C.N.R.S. Marsella

Dr. Jorge Gaspar. Universidad de Lisboa

Dr. André Humbert. Universidad de Nancy 2

Dr. Javier Martín Vide. Universidad de Barcelona

Dr. Eduardo Martínez de Pisón. Universidad Autónoma de Madrid

Dr. Ricardo Méndez Gutiérrez-del Valle. I.E.G. - CSIC, Madrid
Dra. María del Carmen Ocaña. Universidad de Málaga

Dr. Patrick O'Flanaghan. National University of Ireland Cork

Dr. Jorge Olcina Cantos. Universidad de Alicante

Dr. José Ortega Valcárcel. Universidad de Valladolid

Dr. Francisco Quirós Linares. Universidad de Oviedo

Dr. Fernando Rebelo. Universidad de Coimbra

Dra. Edith Rosario Jiménez. Univ. de Guadalajara (México)

Dr. Joan-Eugeni Sánchez. Universidad de Barcelona

Dr. Giorgio Spinelli. Universitá di Roma "La Sapienza" (†)

Dr. Miguel Ángel Troitiño Vinuesa. Univ. Complutense de Madrid

Polígonos está referenciada en los siguientes índices, catálogos y bases de datos:

Latindex

ISOC

Dialnet

DICE

\author{
REDACCIÓN Y ADMINISTRACIÓN \\ Departamento de Geografía y Geología \\ Facultad de Filosofía y Letras \\ Campus de Vegazana \\ Universidad de León \\ 24071 LEÓN \\ Teléfonos: 987/291043-291044 \\ Fax: 987/291877 \\ Normas para los autores / resúmenes de números anteriores: \\ http://www3.unileon.es/dp/dge/publicaciones.php
}

La responsabilidad de las opiniones y los hechos consignados en cada artículo, nota o reseña corresponden exclusivamente a los autores. Los editores no se responsabilizan, en ningún caso, de la credibilidad y autenticidad de los trabajos. En toda reproducción de los materiales publicados, total o parcial, es necesario citar su procedencia

ISSN: 1132-1202

Depósito legal: LE-539-1992

Impreso en León

Editado por los Departamentos de Geografía de las Universidades de León, Salamanca y Valladolid

(C) Del texto: los autores

(C) De la edición: Universidades de León, Salamanca y Valladolid 


\section{POLÍGONOS \\ Revista de Geografía}

Número Monográfico:

\section{EL DEBATE SOBRE EL CAMBIO CLIMÁTICO}

ISSN - 1132-1202

Departamento de Geografía y Geología. Universidad de León Departamento de Geografía. Universidad de Salamanca Departamento de Geografía. Universidad de Valladolid 


\section{POLÍGONOS. Revista de Geografía \\ ISSN 1132-1202 \\ $n^{\circ} 24$}

Número Monográfico

EL DEBATE SOBRE EL CAMBIO CLIMÁTICO

\section{ÍNDICE}

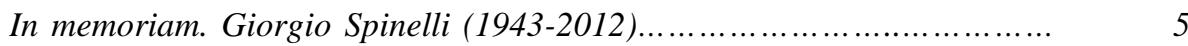

Guillermo CALONGE CANO, Coordinador: Presentación....................... 7

\section{ARTÍCULOS}

Manuel QUIRÓs HERNÁNDEZ: El Cambio Climático y la utilización de las Tecnologías de la Información Geográfica.

Ma Teresa OrTega VillazÁn, Carlos Gabriel Morales RodríGuez y José Luis LABAJO SALAZAR: Aportaciones sobre cambios en las tendencias de las variables climáticas en la Meseta Central Española.

Javier MARTíN-VIDE y María del Carmen MORENO GARCÍA: La difícil determinación de la evolución del número de días de tormenta en España. El caso de Barcelona.

José Miguel Raso NADAL y Juan Carlos PeÑa RABADÁN: La necesidad de soporte a la población de Barcelona ante el impacto potencial de un incremento climático de la temperatura ambiente...

Lorenzo RODRÍGUEZ MAGAZ: Estimaciones objetivas sobre la evolucion regional y global del cambio climático.

José María Redondo VeGA, Javier SANTOS GonZÁLEZ, Blanca GonZÁLEZ GUTIÉRREZ y Amelia GÓMEZ VILLAR: Ejemplos de formas de relieve indicadoras de diferentes paleoclimas en la Cordillera Cantábrica.

\section{NOTAS}

Guillermo CALONGE CANO: Apreciaciones sobre el cambio climático global. Fundamentos e insuficiencias en el contexto de la decisiva contaminación. 


\section{RESEÑAS BIBLIOGRÁFICAS}

Patrice Beghain: Patrimoine, politique et société, La Bibliothèque du ci-

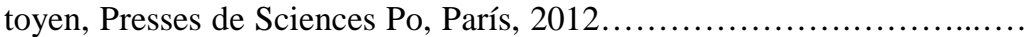

Mar GÉnOva Fuster, Fernando Gómez Manzaneque, Carlos Moral JUARISTI (editores): Los bosques de Gredos a través del tiempo, Ed. Junta de Castilla y León. Consejería de Medio Ambiente. Valladolid, 2009.

James LOVELOCK: La venganza de la Tierra (Por qué la Tierra está rebelándose y como podemos todavía salvar a la humanidad). Editorial Planeta, Barcelona, 2007

José Alberto Rio FERNANDES, Maria Encarnação Beltrão SPOSITO (organizadores): A nova vida do velho centro nas cidades portuguesas e brasileiras. Universidade do Porto-CEGOT, Oporto, 2013 


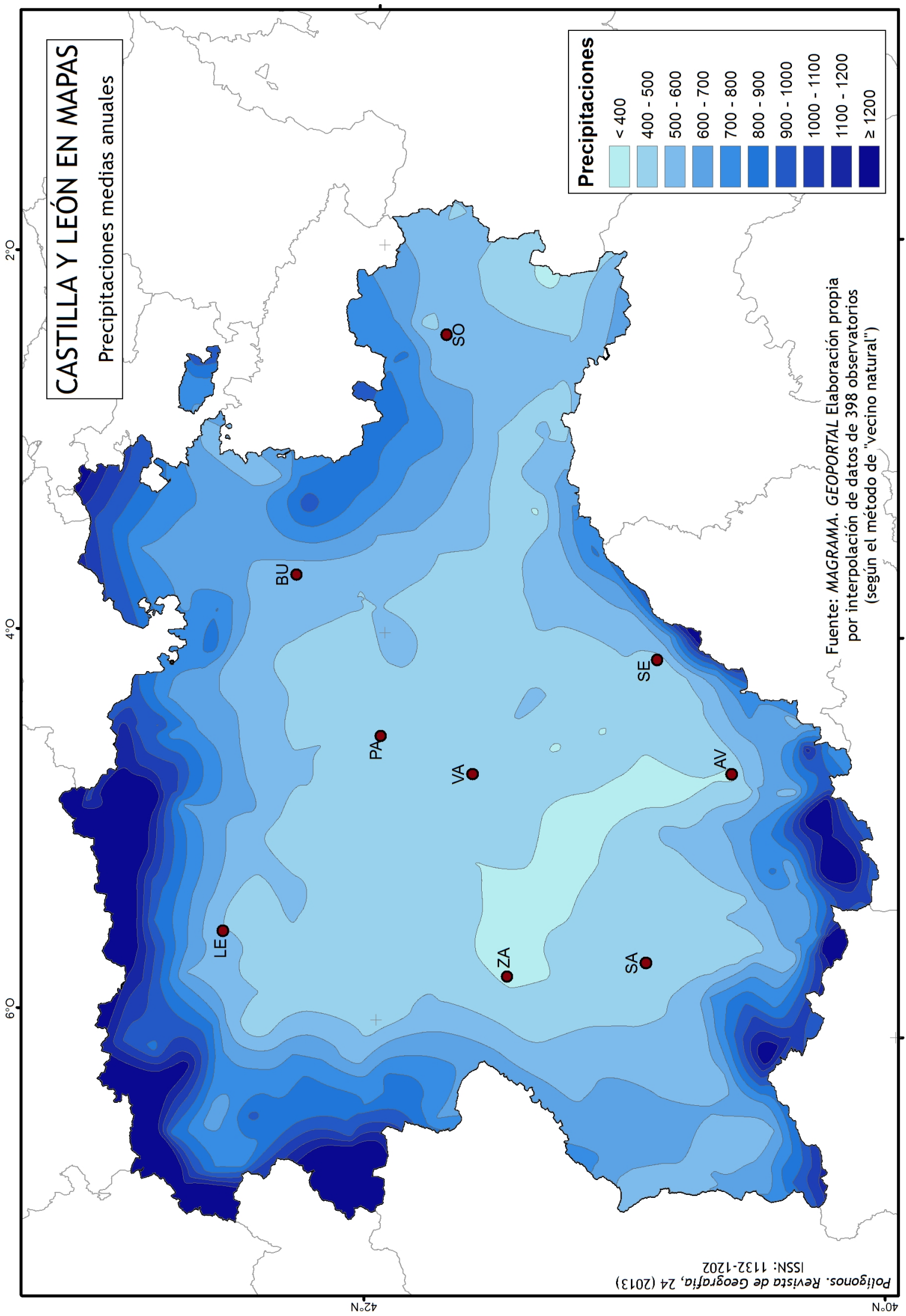




\section{POLÍGONOS}

REVISTA DE GEOGRAFIA

2013

In memoriam. Giorgio Spinelli (1943-2012)

7 Guillermo CALONGECANO, Coordinador

Presentación

\section{ARTÍCULOS}

13 Manuel QUIRÓS HERNÁNDEZ

El Cambio Climático y la utilización de las Tecnologías de la Información Geográfica

$43 \mathrm{M}^{\mathrm{a}}$ Teresa ORTEGA VILLAZÁN, Carlos Gabriel MORALES RODRÍGUEZ y José Luis LABAJO SALAZAR

Aportaciones sobre cambios en las tendencias de las variables climáticas en la Meseta Central Española

77 Javier MARTÍN-VIDE y María del Carmen MORENO GARCÍA

La difícil determinación de la evolución del número de días de tormenta en España. El caso de Barcelona

95 José Miguel RASO NADAL y Juan Carlos PEÑA RABADÁN

La necesidad de soporte a la población de Barcelona ante el impacto potencial de un incremento climático de la temperatura ambiente

\section{Lorenzo RODRÍGUEZMAGAZ}

Estimaciones objetivas sobre la evolucion regional y global del cambio climatico

163 José María REDONDO VEGA, Javier SANTOS GONZÁLEZ, Blanca GONZÁLEZ GUTIÉRREZ y Amelia GÓMEZ VILLAR

Ejemplos de formas de relieve indicadoras de diferentes paleoclimas en la Cordillera Cantábrica

\section{NOTAS}

185 Guillermo CALONGE CANO

Apreciaciones sobre el cambio climático global. Fundamentos e insuficiencias en el contexto de la decisiva contaminación

201 RESEÑAS BIBLIOGRÁFICAS

221 Normas para el envío de originales 